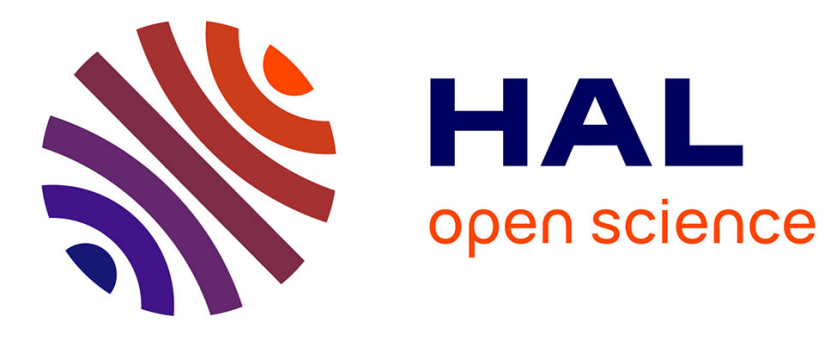

\title{
Obesity in Midlife Hampers Resting and Sensory-Evoked Cerebral Blood Flow in Mice
}

Haleh Soleimanzad, Mireia Montaner, Gaëtan Ternier, Mathilde Lemitre, Jean-Sébastien Silvestre, Nadim Kassis, Paolo Giacobini, Christophe Magnan, Frederic Pain, Hirac Gurden

\section{To cite this version:}

Haleh Soleimanzad, Mireia Montaner, Gaëtan Ternier, Mathilde Lemitre, Jean-Sébastien Silvestre, et al.. Obesity in Midlife Hampers Resting and Sensory-Evoked Cerebral Blood Flow in Mice. Obesity, 2020, 29 (1), pp.150-158. 10.1002/oby.23051 . hal-03048891

\section{HAL Id: hal-03048891 https://hal.science/hal-03048891}

Submitted on 1 Oct 2021

HAL is a multi-disciplinary open access archive for the deposit and dissemination of scientific research documents, whether they are published or not. The documents may come from teaching and research institutions in France or abroad, or from public or private research centers.
L'archive ouverte pluridisciplinaire HAL, est destinée au dépôt et à la diffusion de documents scientifiques de niveau recherche, publiés ou non, émanant des établissements d'enseignement et de recherche français ou étrangers, des laboratoires publics ou privés. 


\title{
Obesity in Midlife Hampers Resting and Sensory-Evoked Cerebral Blood Flow in Mice
}

\author{
Haleh Soleimanzad ${ }^{1 * \#}$, Mireia Montaner ${ }^{l *}$, Gaëtan Ternier $^{2}$, Mathilde Lemitre $^{3}$, Jean-Sébastien Silvestre ${ }^{3}$, \\ Nadim Kassis ${ }^{1}$, Paolo Giacobini ${ }^{2}$, Christophe Magnan ${ }^{1}$, Frédéric Pain $^{4}$, and Hirac Gurden (iD) 1
}

Objective: This study aimed to investigate the effects of a high-fat diet (HFD) and aging on resting and activity-dependent cerebral blood flow (CBF).

Methods: To run a comparison between obese and age-matched control animals, 6-week-old mice were fed either with regular chow or an HFD for 3 months or 8 months. Glucose tolerance and insulin sensitivity were assessed for metabolic phenotyping. Resting and odor-evoked CBF at the microvascular scale in the olfactory bulb (OB) was investigated by multiexposure speckle imaging. Immunolabeling-enabled imaging of solvent-cleared organs was used to analyze vascular density. The ejection fraction was studied by using cardioechography. Olfactory sensitivity was tested by using a buried-food test.

Results: Glucose intolerance and compromised odor-evoked CBF were observed in obese mice in the younger group. Prolonged HFD feeding triggered insulin resistance and stronger impairment in activity-dependent CBF. Aging had a specific negative impact on resting CBF. There was no decrease in vascular density in the $\mathrm{OB}$ of obese mice, although cardiac function was impaired at both ages. In addition, decreased olfactory sensitivity was observed only in the older, middle-aged obese mice.

Conclusions: OB microvasculature in obese mice showed a specific functional feature characterized by impaired sensory-evoked CBF and a specific deleterious effect of aging on resting CBF.

Obesity (2021) 29, 150-158.

\section{Study Importance}

What is already known?

- Obesity is a metabolic condition with higher risk for ischemic stroke.

- Aging affects brain hemodynamics in lean animals.

What does this study add?

- Obesity impairs odor-evoked cerebral blood flow (CBF).

- Aging specifically blunts resting CBF in lean and obese mice.

- Microvascular density does not decrease in the olfactory bulb of obese mice.

\section{Introduction}

The pandemic of obesity is a worldwide health threat; according to the World Health Organization (1), in 2016, more than 1.9 billion adults had overweight, and of these, more than 650 million had obesity. Obesity is a metabolic disease primarily defined as a condition in which excessive fat accumulation in the body adversely affects health (2). It is associated with comorbidities such as type 2 diabetes or cardiovascular disease and it also has a detrimental effect on brain activity (3-4). Because of a lack of energy storage, the brain needs a continuous supply of energy. The necessity of this ongoing energy feeding implies a tight and reliable regulation of cerebral blood flow (CBF) both at rest and during the activated states of cerebral networks (5). Obesity is a metabolic condition causing a higher risk of ischemic stroke in clinics (6) and a higher risk of toxicity along the human blood-brain barrier (7). In addition, rodent models of obesity have repetitively been shown to have worse outcomes after experimental stroke (8-10). Aging has been shown to be an important factor in exacerbating the deleterious effects of obesity on brain function (11-12) and it could be responsible for further disturbing brain hemodynamics of organisms with obesity as they age (13-15). However, the fate of CBF regulation at rest and in response to physiological neuronal activation over the course of high-fat diet (HFD) feeding (i.e., aging superimposed on obesity) remains unexplored.

\footnotetext{
${ }^{1}$ Université de Paris, Unit of Functional and Adaptive Biology (BFA), UMR 8251 CNRS, Paris, France. Correspondence: Hirac Gurden (hirac.gurden@u-paris.fr) ${ }^{2}$ Université de. Lille, INSERM, CHU Lille, U1172 - LilNCog - Lille Neuroscience \& Cognition, Lille, France ${ }^{3}$ Université de Paris, Paris Cardiovascular Research Center (PARCC), INSERM, Paris, France ${ }^{4}$ Université Paris-Saclay, Institut d'Optique Graduate School, CNRS, Laboratoire Charles Fabry, Palaiseau, France.

See Commentary, pg. 17.

*Haleh Soleimanzad and Mireia Montaner contributed equally to this study and should be considered co-first authors.

\#Present address: Institut Physique pour la Médecine, Paris, INSERM U1273, CNRS FRE2031, ESPCI Paris, Paris, France.

(C) 2020 The Obesity Society. Received: 30 July 2020; Accepted: 14 September 2020; Published online 10 November 2020. doi:10.1002/oby.23051
} 
To study neurovascular mechanisms allowing local adaptation of hemodynamics to neuronal activity, sensory cortices in rodents were used as a system model. Odor-evoked neurovascular mechanisms in the main olfactory bulb (OB), the first cerebral structure coding for odors (16), have been studied for a couple of decades. Hemodynamic changes in the $\mathrm{OB}$ have been recorded using several imaging techniques with complementary spatiotemporal resolution (17-18), which made it possible to characterize functional hyperemia (a local increase of CBF triggered by neural activation) and neurovascular coupling mechanisms at different vasculature scales, from pial arteries to single capillaries. In addition, the role of the different cellular entities (19-22) has been determined in odor-evoked functional hyperemia. In the present study, to map vascular activity at the OB surface, we used a full-field optical imaging technique: multiexposure speckle imaging (MESI) (23-25). We recorded CBF at rest and in response to odor presentation in anesthetized obese mice fed a HFD for 3 or 8 months and their age-matched lean controls. We also characterized the metabolic phenotype, vascular density, cardiac function, and olfactory behavior of these obese mice. Our results indicate a specific functional feature of $\mathrm{OB}$ vasculature in obese mice, compared with control mice, that is characterized by impaired odor-evoked CBF and a specific deleterious effect of aging on resting $\mathrm{CBF}$.

\section{Methods}

\section{Animals}

All procedures were approved by the animal care and use committee of the University of Paris (CEEA40, ethical project 7582) in accordance with the European directive 2010/63/UE. Male C57BL/6 mice (received at 5 weeks of age; Janvier Labs) were housed in cages with ad libitum regular food (A04 diet, 2,791 kcal/kg; Safe) and tap water and maintained on a 12-hour light/12-hour dark cycle. Cages were enriched with tunnels. To trigger obesity, mice were given an ad libitum HFD (HF230 diet, 5,317 kcal/kg; Safe) together with regular food starting at 6 weeks of age (after 1 week of acclimatization). Control mice were kept on regular food. According to the HFD duration, mice were divided into two groups, each of them including obese and control mice: 4.5-month-old control mice fed regular food versus 4.5-month-old obese mice fed a HFD for a duration of 3 months (3M group; adult mice) and 9.5-month-old control mice fed regular food versus 9.5-month-old obese mice fed a HFD for a duration of 8 months ( $8 \mathrm{M}$ group; middle-aged mice). Comparing the $8 \mathrm{M}$ group with the $3 \mathrm{M}$ group enabled us to study aging in control and obese mice. A total of 112 mice were used for the six independent experiments. Subgroup composition is indicated in the figure captions (Figures 1-5; also see Supporting Information Table S1).

\section{Metabolic phenotyping}

Body weight and body mass composition. Body weight was measured weekly. Body mass composition was determined by an EchoMRI-900 (Echo Medical Systems).

Oral glucose tolerance test. To evaluate the impact of a HFD on glycemia, oral glucose tolerance tests (OGTT) were performed in 5-hour-fasted mice. A glucose solution $(1 \mathrm{~g} / \mathrm{kg})$ was administrated by oral gavage. Blood glucose was quantified from the tip of the tail vein

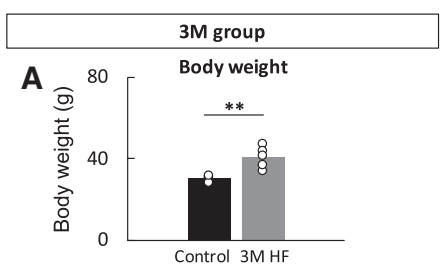

B

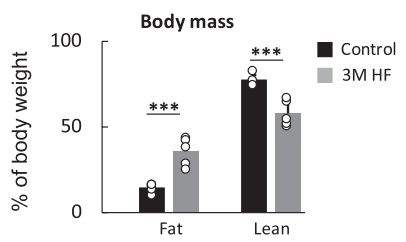

C

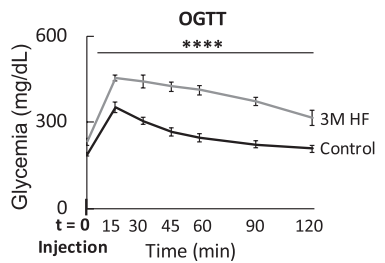

D
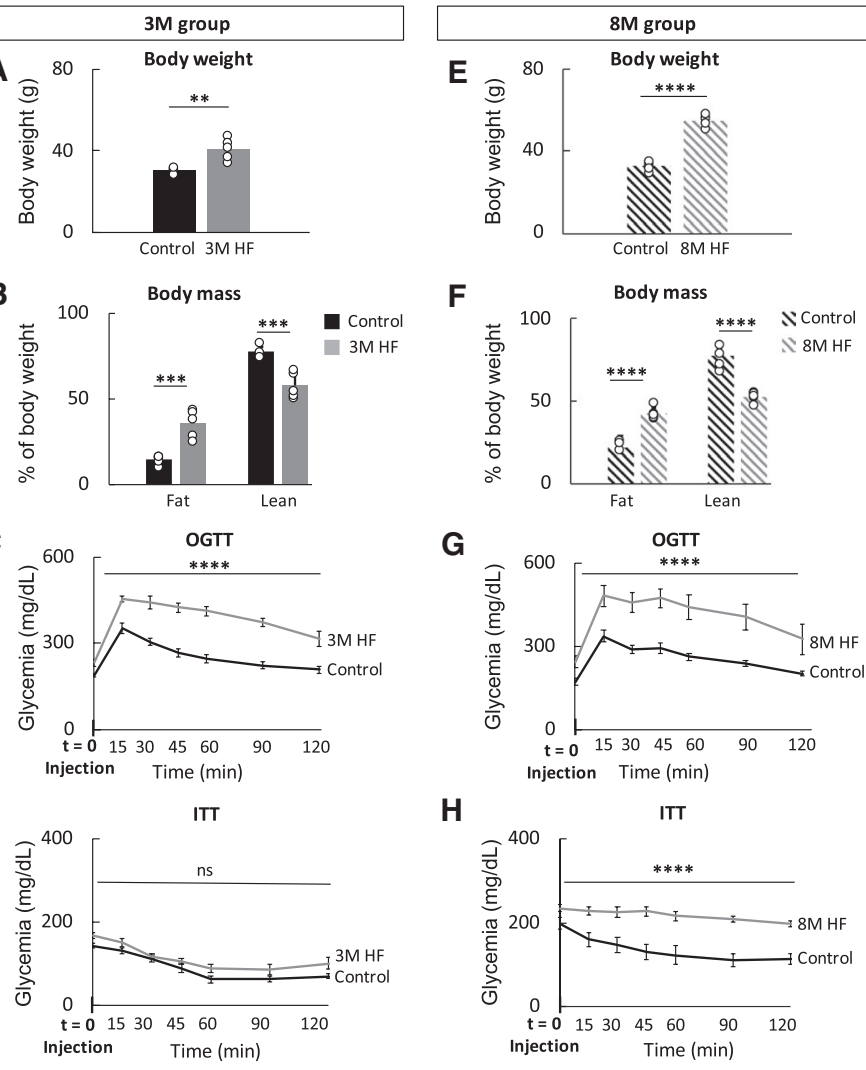

$\mathbf{F}$

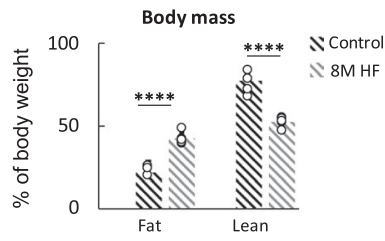

G

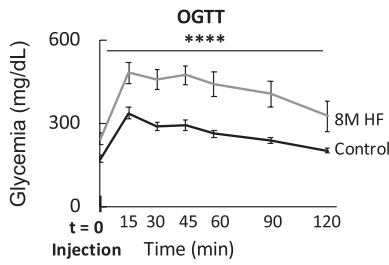

H
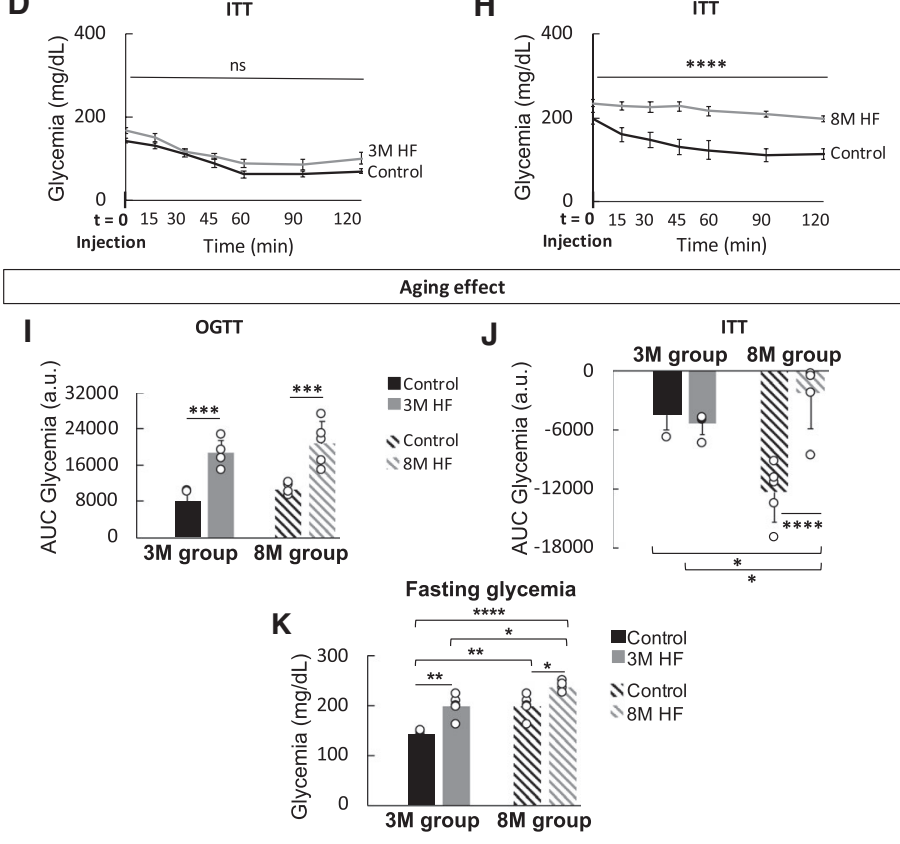

Figure 1 (A-D) Metabolic phenotyping of 4.5-month-old control mice fed regular food and 4.5-month-old obese mice fed a high-fat diet (HF) for a duration of 3 months (3M group; young-adult mice) (left column). (A) Body weight, (B) fat and lean mass, and (C) time course of glycemia during oral glucose tolerance tests (OGTT). Time 0 corresponds to glucose gavage $(1 \mathrm{~g} / \mathrm{kg}$ ). (D) Time course of glycemia during insulin tolerance tests (ITT). Time 0 corresponds to intraperitoneal administration of insulin $(0.75 \mathrm{U} / \mathrm{kg})$. (E-H) Metabolic phenotyping of 9.5-month-old control mice fed regular food and 9.5-month-old obese mice fed a HF for a duration of 8 months (8M group; middle-aged mice) (right column). The right column depicts the same diagram types as the left column. Effects of physiological aging in control mice and HF duration (aging superimposed on obesity) in obese mice were tested and compared. The mean area under the curve (AUC) was obtained by calculating the integral of glycemia values from the plots in panels $\mathrm{C}$ and $\mathrm{G}$ for (I) OGTT; for (J) ITT, plots in panels $\mathrm{D}$ and $\mathrm{H}$ were used. (K) Fasting glycemia. Values are mean $\pm S D ; n=5$ per group (total of 20 mice). Unpaired Student $t$ tests in panels A, B, E, and $\mathrm{F}$ and two-way ANOVA in panels C, $\mathrm{D}, \mathrm{G}$, and $\mathrm{H}$ followed by post hoc Bonferroni tests in panels I, J, and $\mathrm{K} .{ }^{*} P<0.05$, ${ }^{\star \star} P<0.01,{ }^{\star \star \star} P<0.001$, and ${ }^{\star \star \star \star} P<0.0001$. a.u., arbitrary units; ns, not significant. 
with a glucose meter (Glucofix Lector, Menarini Diagnostics). Blood samples were collected from the tail vein to assay plasma insulin with a wide-range Ultra Sensitive Mouse Insulin ELISA Kit (catalog no. 90080; Crystal Chem, Inc.).

Insulin tolerance test. To assess insulin sensitivity, insulin tolerance tests $(0.75 \mathrm{U} / \mathrm{kg})$ were performed in 5 -hour-fasted mice. Mice were given an intraperitoneal injection of insulin $(0.75 \mathrm{U} / \mathrm{kg}$ of body weight; Novo Nordisk), and glycemia was monitored following the same protocol as was used for OGTT.

\section{Multiple-exposure speckle imaging}

Mice were anesthetized intraperitoneally with a cocktail of ketamine $(100 \mathrm{mg} / \mathrm{kg})$ and medetomidine $(0.5 \mathrm{mg} / \mathrm{kg})$. The anesthesia level was adjusted if necessary throughout the experiment, and the temperature was kept at $37 \pm 0.5{ }^{\circ} \mathrm{C}$ using a heating pad. After a craniotomy of $1.5 \mathrm{~mm}^{2}$ centered on the anterior-posterior and medial-lateral stereotaxic coordinates of +4 to $5 \mathrm{~mm}$ from bregma and $1 \mathrm{~mm}$ from midline (26), respectively, imaging was carried out.

$\mathrm{CBF}$ at rest and $\mathrm{CBF}$ in response to olfactory stimulations were determined using an in house-designed MESI imager. The MESI protocol was fully described previously (23) and is illustrated in Supporting Information Figure S1. It relies on the determination of the correlation time $\tau_{\mathrm{c}}$ of scatterers (i.e., red blood cells) using multiple exposure acquisitions. The inverse correlation times derived from MESI have been shown to be robust metrics of blood flow changes (27). For simplicity, it will be referred in this article as the "CBF index." Optimized procedures to derive CBF-index maps from MESI have been described in detail previously (23). Briefly, at rest, CBF-index values were obtained for each pixel as the absolute inverse correlation time and averaged during 12 seconds of the baseline recordings for each mouse. Olfactory stimulations were obtained by presentation of a pure odor diluted in mineral oil and placed in one vial of a custom-designed olfactometer. A simulation trial consists of $12 \mathrm{sec}-$ onds of the baseline state (air flow) and 12 seconds of odorant stimulation, followed by 24 seconds of air flow to clean up the system. One experiment consisted of the recording of five trials with hexanal $5 \%$ or isoamyl acetate $5 \%$ as odors. Stimulations with two odorants were interleaved randomly to prevent habituation. Following stimulation, the relative changes in the $\mathrm{CBF}$ index were calculated pixelwise as the percentage change relative to the baseline CBF index. The average values obtained during the baseline state were subtracted from the average value achieved during stimulation, revealing the locations where $\mathrm{CBF}$ had changed during the stimulation. This difference was then divided by baseline values to obtain a variation in the percentage. Values of five trials were averaged.

\section{Immunolabeling-enabled imaging of solvent-cleared organs}

Mice were perfused transcardially with PBS one time for a duration of 5 minutes and then with $4 \%$ paraformaldehyde for a duration of 5 more minutes. Brains were postfixed in $4 \%$ paraformaldehyde overnight at $4{ }^{\circ} \mathrm{C}$ and were then then submerged in sucrose. For the analysis of laminin-stained mouse OB (illustrated in Supporting Information Figure S2), we used the immunolabeling-enabled imaging of solvent-cleared organs (iDISCO) protocol from Renier et al. (2014) (28), with minor adjustments. The primary antibody used was rabbit anti-laminin antibody $(1: 1,000$, catalog no. ab11575; Abcam). The secondary antibody used was donkey anti-rabbit AlexaFluor 568 (1:500; Thermo Fisher Scientific).

Light-sheet imaging. Imaging was performed on the Ultramicroscope (LaVision Biotec) light-sheet microscope available at the BioImaging Center of Lille. The field and depth were set to cover the two bulbs, and the z-step was set to $2 \mu \mathrm{m}$.

Data extraction and analysis. Data from light-sheet imaging were first converted to the Imaris file format. The Surfaces tool of Imaris 9.3 (Bitplane) was used to create surfaces on the basis of the laminin signal, with a fixed threshold for detection, in a region of interest (ROI) set to cover a $500 \mu \mathrm{m}$-thick volume in the center of the second part of the OB (+4.28 mm anterior to bregma (26)). The resulting surface was used as a mask to create a new channel of fixed intensity, which was then extracted as a sequence of 2- $\mu \mathrm{m}$ slices and further processed in Fiji. A macro relying on the particle-analysis tool was used on the stacks to detect, classify, and segment the vessels on the basis of their cross-sectional area (5-10 pixels squared $\left[\mathrm{px}^{2}\right], 10-20 \mathrm{px}^{2}, 20-50 \mathrm{px}^{2}$, and $\left.>50 \mathrm{px}^{2}\right)$. The relative frequency of each class was calculated for the different groups as follows: frequency =number of particles in the class/total number of particles $>5 \mathrm{px}^{2}$ (Supporting Information Figure S2).

\section{Echocardiography}

Left ventricular (LV) function was assessed by transthoracic echocardiography with a VEVO2100 Biomicroscope (VisualSonics). Mice were anesthetized with isoflurane (1.5\% in air) and monitored for respiratory frequency and temperature. Parasternal long-axis views of the left ventricle were obtained with a $40-\mathrm{MHz}$ ultrasound probe (MS550D) at a frame rate between 180 and 240 frames per second. Measurements of LV end-diastolic volume (LVEDV) and LV end-systolic volume (LVESV) were performed offline (Cardio Package/VEVO2100 software; VisualSonics). The ejection fraction (EF) percentage was calculated by using the following formula: EF percentage $=([$ LVEDV - LVESV $] /$ LVEDV $) \times 100$.

\section{Real-time quantitative polymerase chain reaction}

Total RNA was isolated using RNeasy Lipid Tissue mini kit (Qiagen). Real-time quantitative polymerase chain reaction (PCR) was carried out in a LightCycler 480 detection system (Roche) using the LightCycler FastStart DNA Master plus SYBR Green I kit (Roche). The primers were derived from mouse sequences. We targeted the glial fibrillary acidic protein (GFAP) mRNA expressed in astrocytes (GFAP_mAS agccgtccagagggaacta/GFAP_mS agccgtccagagggaacta) normalized against the mean of two reference house-keeping genes (TBP_m_S ggggagctgtgatgtgaagt/TBP_m_AS ccaggaaataattctggctca; rpL19_m_S gggcaggcatatgggcata/rpL19_m_AS ggcggtcaatcttcttggatt). TATAbinding protein and ribosomal protein L19 were previously used as reference genes in the mouse OB (29) and the human neocortex (30).

\section{Buried-food test}

Because rodents naturally use olfactory cues for food foraging, we used the buried-food test as a general and reliable estimation of olfactory sensitivity $(29,31)$.

Individual mice underwent two consecutive days (day 1 and day 2) of odorized-food familiarization to prevent neophobia during the test. One gram of chocolate-flavored cookie was placed in the home 

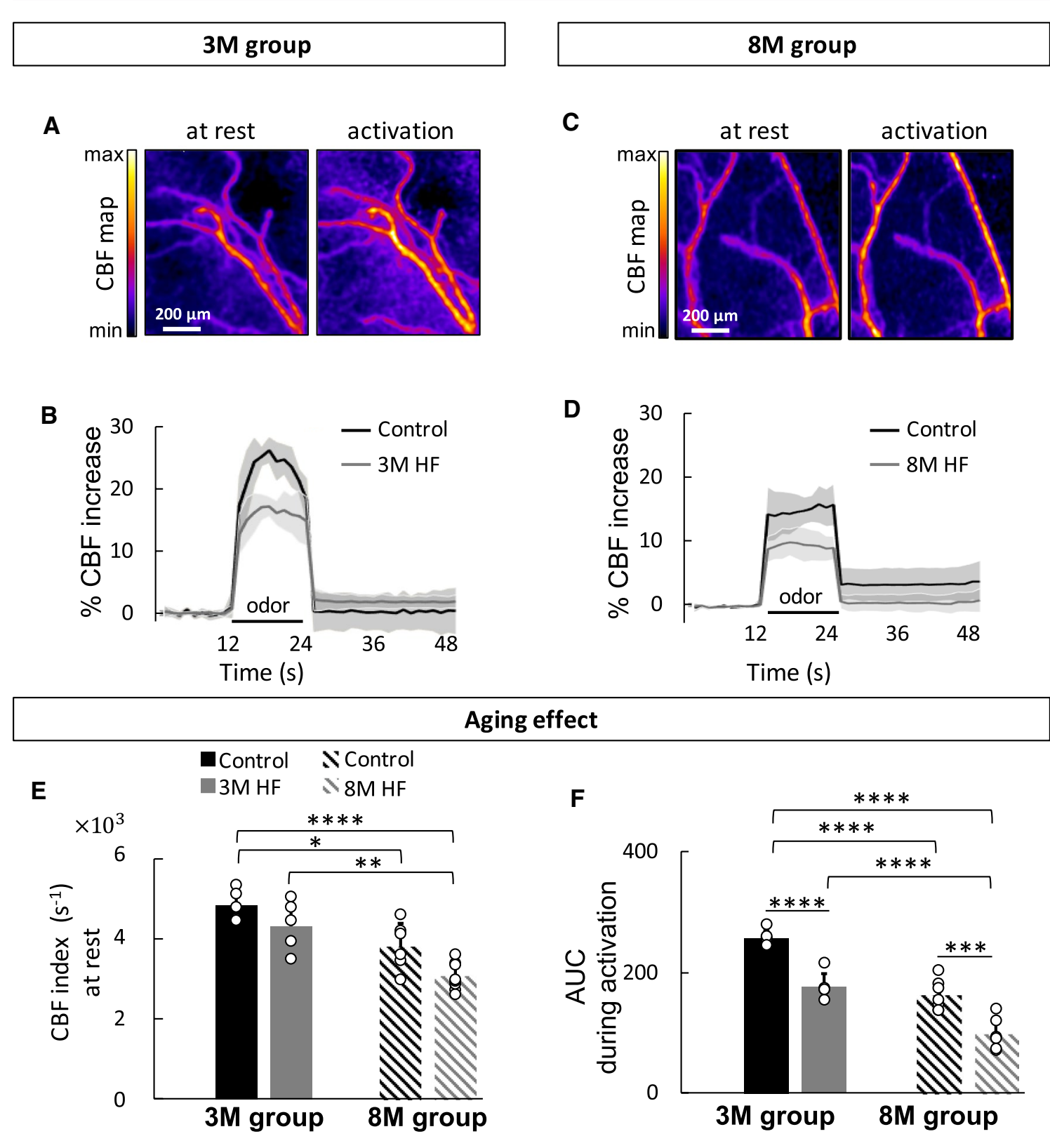

Figure 2 In vivo multiexposure speckle imaging (MESI) data at rest and under sensory activation in the olfactory bulb (OB) of 4.5-month-old control mice fed regular food and 4.5-month-old obese mice fed a high-fat diet (HF) for a duration of 3 months (3M group; young-adult mice) (left column). (A) Representative cerebral blood flow (CBF) maps at rest and under odor stimulation with $5 \%$ hexanal recorded on the dorsal OB of HF-fed mice. (B) Mean relative changes in CBF during olfactory stimulation with hexanal $5 \%$ for control and HF-fed mice (curves in transparency define the SD; $n=5$ per group, total of 10 mice). (C-D) In vivo MESI data at rest and under sensory activation in the OB of 9.5-month-old control mice fed regular food and 9.5-month-old obese mice fed a HF for a duration of 8 months (8M group; middle-aged mice) (right column). Represented data are of the same types depicted in the left column, with $n=6$ for control mice and $n=8$ for HF-fed mice (total of 14 mice). Effects of physiological aging in control mice and HF duration (aging superimposed on obesity) in obese mice were tested and compared. (E) CBF index at rest in the same control and HF-fed mice described in panels B and D (two-way ANOVA followed by post hoc Bonferroni test). (F) The area under the curve (AUC) was derived from the plots in panels $B$ and $D$ for the $3 \mathrm{M}$ and $8 \mathrm{M}$ groups to further probe the aging effect. Values are mean $\pm \mathrm{SD}\left(n=5\right.$ per $3 \mathrm{M}$ group and $n=6-8$ per $8 \mathrm{M}$ group; two-way ANOVA followed by post hoc Bonferroni test). ${ }^{*} P<0.05$, ${ }^{\star \star} P<0.01,{ }^{\star \star \star} P<0.001$, and ${ }^{\star \star \star \star} P<0.0001$. max, maximum; min, minimum.

cage in a location visible to the animal before the night shift and left overnight. The next morning, total food consumption was inspected. The day before the test day (day 3), mice were deprived of food overnight.
The test was performed on day 4 in a test cage containing $5 \mathrm{~cm}$ of fresh mouse bedding and equipped with a video camera. A piece of chocolate-flavored cookie was buried $2 \mathrm{~cm}$ beneath the surface of the bedding in a random corner of the cage. Latency time was defined as the amount 

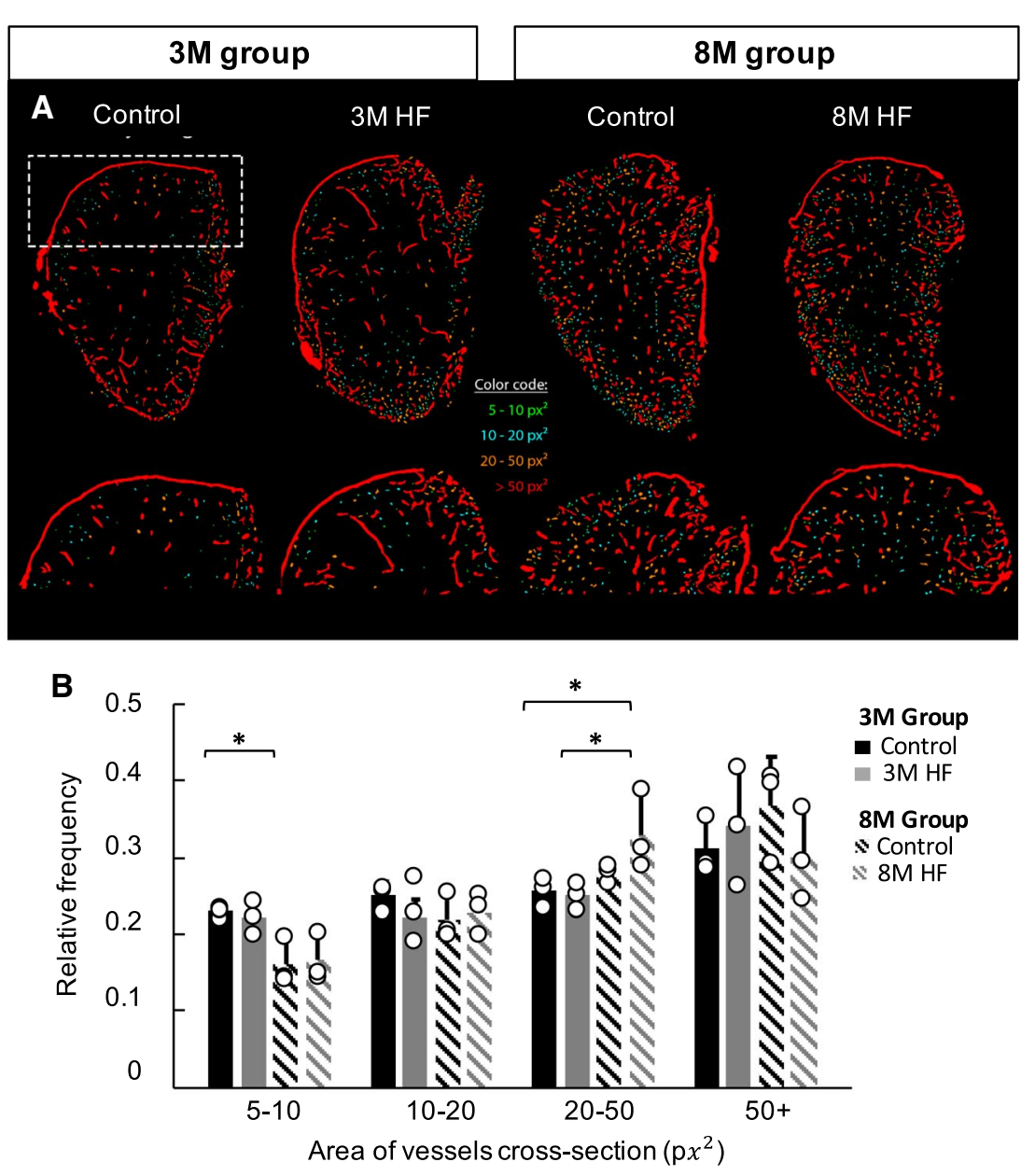

Figure 3 Detection, classification, and analysis of the olfactory bulb (OB) vessels. (A) Vessels detected by the particle analysis in a 500- $\mu \mathrm{m}$-thick subvolume of the OB were classified according to their cross-sectional area. The first row shows representative pseudocolored reslices of the processed stacks. The white box highlights the dorsal layer of the $\mathrm{OB}$, which is magnified in the second raw layer for each reslice, and represents the depth reachable by the laser speckle contrast imaging analysis. Green and blue particles correspond to the classes of vessels expected to be capillaries and arterioles/venules, respectively, whereas orange and red particles depict larger vessels consisting of veins and arteries. (B) The segmentation of the vessels revealed the distribution of the vessels in the different groups and conditions. Statistical analysis of the frequencies for each class notably points to a reduction in capillaries with age in control animals and an increase in arteries and veins with age in high-fat diet (HF)-fed animals (mean $\pm \mathrm{SD}, n=3$ per group, total of 12 mice, two-way ANOVA test followed by post hoc Fisher least significant difference test). ${ }^{\star} P<0.05$. $\mathrm{px}^{2}$, pixels squared.

of time between the introduction of the mouse in the cage and retrieval of the cookie after foraging. Each mouse was tested twice (60-minute interval) and had to find the cookie in both trials to be included in statistics (mean latency of Two trials). Latency was manually counted using Kinovea.

\section{Statistical analysis}

Results were expressed as the mean \pm SD. Statistical analysis was performed in XLSTAT or GraphPad Prism. The Shapiro-Milk test was used to assess data normality. Then, as indicated in each figure caption (Figures 1-5), intergroup comparison was performed using parametric statistics, either through an unpaired Student $t$ test or through twoway ANOVA followed by an appropriate post hoc test for two-by-two comparisons. Values were considered statistically different if the $P$ value was $<0.05$, except for in multiple comparisons in which $P$ was adjusted as required. Levels of significance are indicated by $* P<0.05$, $* * P<0.01, * * * P<0.001$, and $* * * * P<0.0001$.

\section{Results}

\section{The obesity phenotype is worsened in HFD-fed mice as they age}

Three months of HFD feeding caused body weight gain in HFD-fed mice $(+40 \%)$ compared with age-matched young-adult control mice (3M group, Figure $1 \mathrm{~A} ; P=0.0002$ ). Overweight was accompanied by a 


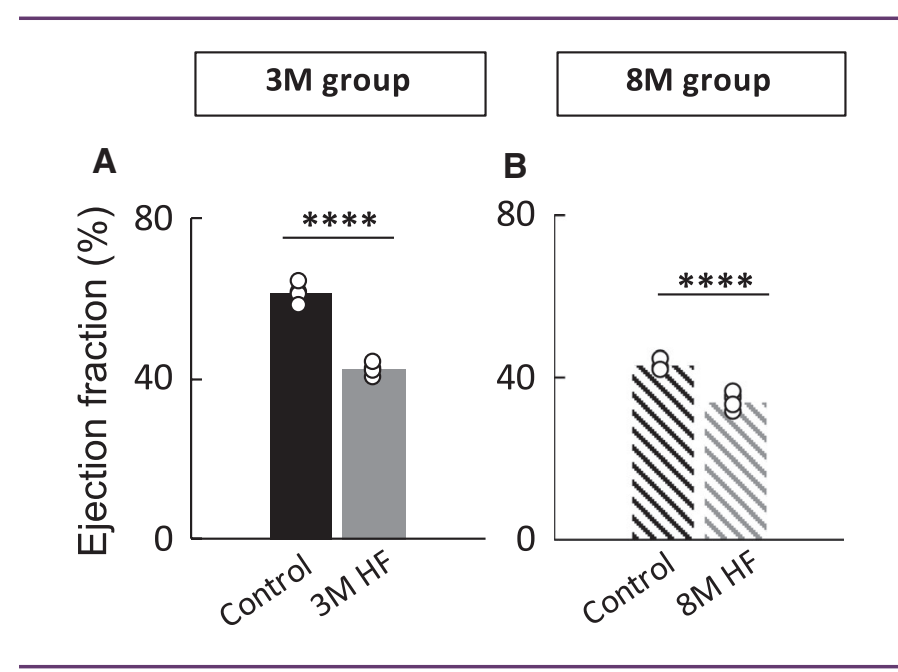

Figure 4 Cardiac ejection fraction in high-fat diet (HF)-fed mice compared with agematched control mice. (A) Young-adult (4.5-month-old) mice fed for a duration of 3 months (3M group): control (black column) versus HF-fed mice (gray column); $n=4$ mice per group. (B) Middle-aged (9.5-month-old) mice fed for a duration of 8 months (8M group): control (column with black dashed lines) versus HF-fed mice (column with gray dashed lines); $n=5$ mice per group. Values are mean $\pm S D$; unpaired Student $t$ tests. ${ }^{* \star *} P<0.0001$.

significant increase in fat mass $(+147 \%)$ to the detriment of lean mass $(-25 \%)$ in $3 \mathrm{M}$ HFD-fed mice compared with control mice (Figure 1B; $P=0.0006$ and 0.0007 , respectively). OGTT results showed glucose intolerance in HFD-fed mice compared with control mice (Figure 1C; $P<0.0001)$. This intolerance was associated with increased insulinemia during the test and with the loss of the glucose-dependent insulin secretion response (Supporting Information Figure S3). 3M HFD-fed mice were sensitive to insulin (Figure 1J), although they had already displayed fasted hyperglycemia compared with $3 \mathrm{M}$ controls $(+40 \%$, Figure $1 \mathrm{~K} ; P=0.003)$.

Eight months of HFD feeding further increased body weight gain in HFD-fed mice (+61\%) compared with age-matched middle-aged control mice (8M group, Figure 1E; $P<0.0001)$. Fat mass was severely increased $(+165 \%)$, and lean mass $(-33 \%)$ was decreased (Figure $1 \mathrm{~F}$; $P<0.0001)$. OGTT revealed that $8 \mathrm{M}$ HFD-fed mice were glucose intolerant compared with control mice (Figure $1 \mathrm{G} ; P<0.0001$ ). This intolerance was associated with sustained increased insulinemia during the test and with the loss of the glucose-dependent insulin secretion response (Supporting Information Figure S3). Importantly, 8M HFDfed mice were insensitive to insulin (Figure $1 \mathrm{~J} ; P<0.0001$ ) and displayed hyperglycemia ( $+20 \%$, Figure $1 \mathrm{~K} ; P=0.048)$.

We further probed aging effects on metabolic parameters by representing the area under the curve (AUC) of metabolic data from the $3 \mathrm{M}$ and $8 \mathrm{M}$ groups. There was no effect of physiological aging ( $8 \mathrm{M}$ vs. $3 \mathrm{M}$ control mice) or HFD duration (aging superimposed on obesity, $8 \mathrm{M}$ vs. 3M HFD-fed mice) on glucose tolerance (Figure 1I). We found a significant effect of HFD duration (Figure $1 \mathrm{~J} ; P=0.0001$ ) and a slight effect of combined age and diet (8M HFD-fed mice vs. 3M control mice, Figure $1 \mathrm{~J} ; P=0.036$ ) but found no effect of aging on insulin sensitivity. In addition, aging, HFD duration, and combined age and diet had a significant effect on fasted hyperglycemia (Figure $1 \mathrm{~K} ; P=0.0027$, 0.048 , and 0.0001 , respectively).

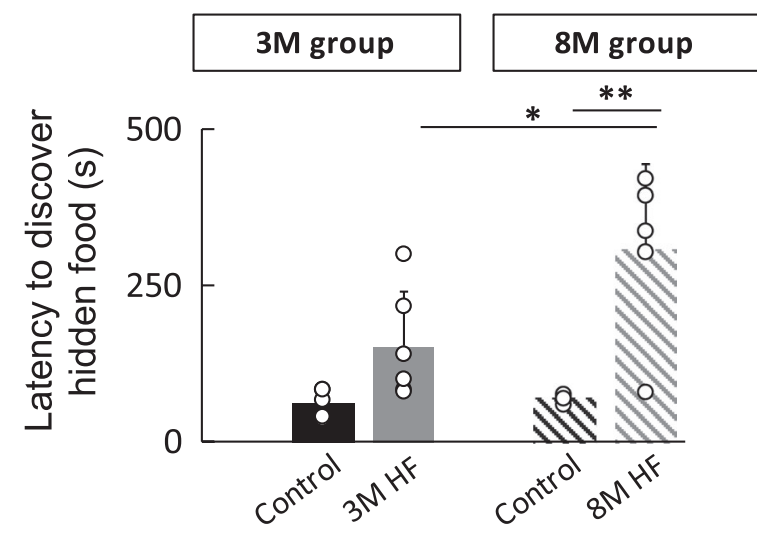

Figure $\mathbf{5}$ Latency in olfactory foraging to uncover an aromatized food pellet. Food discovery time was measured during a buried-food test after an overnight fast in each group. Values are mean \pm SD (control and high-fat diet [HF]-fed mice: $n=5$ and $n=6$, respectively, for the 4.5-month-old mice fed over 3 months [3M group]; $n=4$ and $n=5$ respectively for 9.5-month-old mice fed over 8 months [8M group]). Two-way ANOVA followed by post hoc Bonferroni test. ${ }^{*} P<0.05$ and ${ }^{* \star} P<0.01$.

\section{Odor-evoked CBF is decreased in obese mice at both ages compared with control mice, and resting $\mathrm{CBF}$ is impaired with aging in control and obese mice}

The CBF index was derived from MESI data to evaluate blood flow changes in HFD-fed mice versus control mice at rest and under odorevoked activation. Figure $2 \mathrm{~A}$ and $2 \mathrm{C}$ present $\mathrm{CBF}$-index maps at rest and during olfactory stimulation in the OB of HFD-fed mice from the $3 \mathrm{M}$ group and $8 \mathrm{M}$ group, respectively. MESI enabled the visualization of the complex vascular architecture at the surface of the $\mathrm{OB}$ with a network of microvessels (mean diameter of $25 \pm 8 \mu \mathrm{m}$ ) (23). The size $(<20 \mu \mathrm{m})$ and the blood speed $(3-15 \mathrm{~mm} / \mathrm{s})$ within these vessels match the arteriolar compartment. In both groups, there was a significant shift toward higher CBF indexes during olfactory stimulation compared with rest. Further comparison of the CBF index between control and HFD-fed mice during odor stimulation (Figure 2B and 2D) was performed in ROIs defined over regions in the blood vessels (Supporting Information Figure S1C). For all groups, a clear and transient increase in CBF was observed in response to a short puff of odorant. In the $3 \mathrm{M}$ group, the odor-evoked increase in $\mathrm{CBF}$ in $\mathrm{HFD}$-fed mice was lower than that of control mice (Figure $2 \mathrm{~B},+17 \%$ vs. $+26 \%$ respectively; $P<0.0001$ ). In the $8 \mathrm{M}$ group, the odor-evoked increase in CBF in the HFD-fed mice was also lower than that of control mice (Figure 2D, $+9 \%$ vs. $+15 \%$ respectively; $P<0.001)$. These data showed a significant decrease in odor-evoked CBF in the OB of HFD-fed mice compared with agematched control mice.

To quantify resting $\mathrm{CBF}$, the $\mathrm{CBF}$ index at rest was calculated in vascular ROIs. Age and diet significantly impacted resting CBF (Figure 2E; $P<0.001)$ : resting $\mathrm{CBF}$ decreased with age in control mice $(P=0.02)$ and with HFD duration in obese mice $(P=0.001)$. In the $3 \mathrm{M}$ and $8 \mathrm{M}$ groups, the average resting CBF of HFD-fed mice was not different from that of control mice (3M group: $P=0.7 ; 8 \mathrm{M}$ group: $P=0.06$ ). Thus, there was a specific deleterious effect of aging, in control as well as obese mice, on resting $\mathrm{CBF}$. 
To further quantify hemodynamic responses to sensory stimulation and their changes with aging, the AUC (Figure 2F) was derived from odorevoked CBF values in Figure 2B and 2D. The AUC decreased with age in control mice $(P<0.0001)$, with HFD duration in obese mice $(P<0.0001)$, and with age and diet combined (3M control mice vs. $8 \mathrm{M}$ HFD-fed mice; $P<0.0001)$. Very interestingly, we also observed that the AUC of $3 \mathrm{M}$ HFD-fed mice was not different from that of $8 \mathrm{M}$ control mice $(P>0.05$; i.e., odor-evoked $\mathrm{CBF}$ in young-adult HFD-fed mice was equal to that of middle-aged controls); this could indicate that functional hyperemia in HFD-fed mice is impaired by premature aging mechanisms.

\section{HFD and aging modulate vascular network architecture}

In order to investigate potential changes in the vasculature of the $\mathrm{OB}$ in the $3 \mathrm{M}$ and $8 \mathrm{M}$ groups, we performed whole-mount immunohistochemistry using the iDISCO tissue-clearing method coupled with light-sheet microscopy imaging (methods illustrated in Supporting Information Figure S2). Vasculature was immunostained using an antibody against laminin, which allows efficient labeling of both large and small vessels. The detected vessels were then ordered in classes depending on their size and expected nature: 5 to $10 \mathrm{px}^{2}$ (capillaries), 10 to $20 \mathrm{px}^{2}$ (arterioles, venules), 20 to $50 \mathrm{px}^{2}$ (arterioles, pial arteries, veins), and $>50 \mathrm{px}^{2}$ (pial arteries and veins). The frequency of each class was determined for the four groups (Figure 3A).

Our analysis (Figure 3B) showed a significant reduction with age in the frequency of the first (smallest) class of vessels (5-10 $\mathrm{px}^{2}$ ) for control mice ( $3 \mathrm{M}$ vs. $8 \mathrm{M}$ mice; $P=0.04$ ) but did not show a significant reduction for HFD-fed mice. The third class of vessels (20-50 $\left.\mathrm{px}^{2}\right)$ notably revealed an increased frequency with HFD duration ( $3 \mathrm{M}$ vs. $8 \mathrm{M}$ mice; $P=0.02$ ), but this was not present in control mice. Overall, we did not detect any changes in the frequencies of the four classes between HFD and control mice of the same age (Figure 3B). These results indicate that impaired $\mathrm{CBF}$ in obese mice is independent of changes in vascular density.

\section{Cardiac function is impaired in HFD-fed mice compared with age-matched control mice}

The processes involved in the regulation of resting CBF are multifaceted and include a constant blood supply from the heart. Because we observed a decreased resting CBF in our groups, we monitored cardiac function (Figure 4). The EF was reduced in HFD-fed mice compared with control mice in the $3 \mathrm{M}$ group $(P<0.0001)$ and was also reduced in older HFD-fed mice (8M group; $P<0.0001)$.

\section{Prolonged HFD triggers a decrease in olfactory sensitivity}

We performed a buried-food test to evaluate the effect of the HFD on olfactory behavior (Figure 5). HFD-fed mice spent more time to find the hidden cookie than control mice in the $8 \mathrm{M}$ group $(P=0.004)$, but this difference was not significant in the $3 \mathrm{M}$ group $(P=0.56)$. Although the latency to uncover the cookie for control mice was the same for the $3 \mathrm{M}$ and $8 \mathrm{M}$ control groups, the latency for HFD-fed mice increased with HFD duration $(P=0.049)$. These data show that HFD duration, but not physiological aging, triggered a decrease in olfactory sensitivity.

\section{Discussion}

In this study, we analyzed the hemodynamic profiles of $3 \mathrm{M}$ and $8 \mathrm{M}$ HFD-fed mice and compared them with their age-matched lean controls. We also analyzed aging effects by comparing middle-aged mice with young-adult mice. A summary of all results is presented in Supporting Information Table S1.

We found that a HFD $(60 \% \mathrm{kcal}$ from fat) triggered obesity, with a significant gain in weight due to an increase in fat mass at both ages. Young-adult obese mice had already presented metabolic defects such as fasted hyperglycemia and glucose intolerance. These abnormalities were largely amplified in middle-aged mice, which had a shift from insulin sensitivity to insulin resistance (IR). Previous studies have reported similar metabolic defects with a comparable HFD (8-9). Our results clearly showed a deleterious impact of obesity on odor-evoked CBF (impairment for young-adult and middle-aged obese mice compared with their age-matched lean controls). Physiological aging as well as HFD duration also induced a decrease in odor-evoked CBF. These results revealing neurovascular uncoupling are in line with previous observations showing (i) a decrease in visually evoked functional hyperemia recorded by functional magnetic resonance imaging in people with obesity and type 2 diabetes (32) and in lean, older individuals (33) in clinics and (ii) a decrease in sensory-evoked CBF in the somatosensory cortex of young-adult and older obese rodents (10,14-15).

To our knowledge, this is the first time that decreased levels of resting CBF with aging in a defined brain structure in control and obese mice have been evidenced. In addition, we observed a very significant decrease in the cardiac EF, a common sign of cardiomyopathy in human and rodent obesity (34), in our obese mice. We postulate that there could be a significant impact of cardiac dysfunction on resting $\mathrm{CBF}$, resulting from the failure of the regulatory systems of neurovascular tone in the $\mathrm{OB}$ of obese mice. Indeed, it has been shown in the rat $\mathrm{OB}$ that stimulation of sympathetic adrenergic nerves induces a decrease in resting CBF (35). Interestingly, resting CBF changes induced by noradrenalin were larger in the $\mathrm{OB}$ of older rats than in the $\mathrm{OB}$ of young-adult rats (35), showing a loss of neuromodulatory control of resting $\mathrm{CBF}$ in the $\mathrm{OB}$ with aging. Whether this noradrenergic dysregulation in the $\mathrm{OB}$ is present in obese mice is currently unknown. In this context, future studies will be focused on the description of molecular mechanisms involved in CBF impairment; molecular pathways implicated in endothelial dysfunction, mitochondrial oxidative stress, and neuroinflammation have been shown to be causal in age-related neurovascular uncoupling in mice (36) and will have to be studied in our HFD model. Of note, it has recently been shown that deficiency in transcription nuclear factor erythroid-2-related factor 2, a prosurvival transcription factor with antioxidant properties, is implicated in neurovascular dysfunction in both aging and obesity (37).

One explanation of compromised functional hyperemia in HFD-fed mice could be anatomical changes in the vasculature. In order to get inside the vascular anatomy in the $\mathrm{OB}$, we estimated the vascular network density using iDISCO for the three-dimensional analysis of laminin-labeled endothelial cells (28). Our results did not reveal any significant changes in the vascular density in obese mice compared with control mice. On the contrary, middle-aged obese mice showed increased density of the pial and parenchymal arteriolar compartment compared with young-adult obese mice, which could potentially compensate for the decrease of capillaries with aging that we observed in middle-aged versus young-adult control mice. This counterintuitive protective effect has to be discussed in light of controversial data published about the density of capillaries under a HFD: on one hand, a HFD (15) and aging (38) have been shown to have a detrimental effect 
on capillary density, but on the other hand, the endothelial network has been shown to remain stable in the hippocampus of long-term HFDfed rats (39) or mice (13). The use of different vascular quantitation techniques as well as the quality and duration of HFD can be causal in these discrepancies. Changes in vessel densities that we detected in aging and obesity in our HFD model could be due to modifications in endothelial-cell proliferation. In the context of obesity and diabetes, data about angiogenesis are mainly centered on the adipose tissue and the heart and are sparse concerning the brain. It has been shown in the heart of patients with obesity and diabetes that capillaries and pericytes are destabilized and the ratio of pro- to antiangiotic factors is decreased, causing a loss in the renewal capacity of peripheral blood vessels (40). Additionally, in diabetic patients, cardiac micro- and macrovessels present similarly deficient regeneration (41). Some of these mechanisms involved in the loss of endothelial proliferation in response to angiogenic stimuli have also been described in the brain during aging and obesity (42), showing a possible convergence of pathological pathways in these conditions. We can conclude that functional hyperemia was impaired in the OB of young-adult and middle-aged obese mice, whereas the density of the vascular network was globally intact.

The global metabolic state of young-adult obese mice deteriorated, but insulin sensitivity, a major metabolic factor for proper brain function, was preserved. By contrast, middle-aged obese mice presented IR, increased levels of GFAP transcripts in the OB (Supporting Information Figure S4), and deficits in olfactory foraging. Thus, the mechanistic link between a deteriorated metabolic state and impaired neurovascular coupling and behavior in middle-aged obese mice could be the emergence of IR. Indeed, obesity and aging are the two major risk factors for IR occurrence in humans (43). IR negatively impacts cardiovascular activity and brain perfusion in middle-aged human adults (44). Deficiency of insulin-like growth factor-1 (IGF-1), an insulin-like peptide, has been shown to impair neurovascular coupling in lean mice, a mechanism involving changes in astrocytic signaling (45). Insulin has been shown to regulate $\mathrm{OB}$ activity (46) and promote olfactory perception in humans (47). Consequently, regarding our findings and the literature, the next experimental step would be to further describe the role of insulin in neurovascular coupling in obese mice.

Because aging was shown to further compromise vascular activity in obese mice $(13,15)$, we asked whether it could trigger hemodynamic impairment per se or in combination with obesity. We first observed that resting $\mathrm{CBF}$ and odor-evoked $\mathrm{CBF}$ were significantly decreased in middle-aged control mice compared with young-adult control mice, as previously reported in the barrel cortex $(15,38)$. Vascular density in the $\mathrm{OB}$ was also decreased with aging, conforming with previous results (48). Such an age-related compromised anatomical-functional feature of the microvasculature in the OB has been repetitively demonstrated in other brain regions previously $(38,49-50)$. However, middle-aged control mice with decreased capillary density in the OB did not show impairment in olfactory foraging. Thus, impaired functional hyperemia with apparent healthy aging in mice can be considered as an early sign of future deleterious effects on brain function, as has been suggested in humans (51-52). Very interestingly, we did not find any functional test through which we could distinguish middle-aged control mice from young-adult obese mice (Supporting Information Table S1). These results could indicate a premature age-related neurovascular defect in young-adult obese mice. Indeed, the brains of middle-aged human adults may age prematurely if the individuals have obesity (51). Protection of the cerebral microvasculature could be a biomedical strategy to prevent brain dysfunction in people with obesity as they age.
One limitation of our study is the animals' age. In middle-aged lean mice (at $25 \%$ of the potential lifespan for laboratory mice), we showed that $\mathrm{CBF}$ was already jeopardized, whereas metabolism and behavior were intact, leading to a misleadingly healthy phenotype. Characterizing the anatomical-functional features of the brain vasculature in older mice will be the subject of experiments in the near future, which will provide insight for geroscience (53).

In conclusion, obesity-related vascular dysfunction in the $\mathrm{OB}$ of our HFD model concerns both resting and sensory-evoked CBF regulation and it is independent of blood-vessel density. We also provided new evidence in support of the deleterious effect of (i) aging on resting CBF and (ii) aging in obese mice, with prolonged HFD feeding, on brain hemodynamics. We propose that IR could be responsible for compromised functional hyperemia and impaired behavioral outcomes in obese mice as they age. $\mathbf{O}$

\section{Acknowledgments}

We thank Aurélie Djemat, Magguy Boa, Ludovic Maingault, Angélique Dauvin, Florianne Michel, Daniel Quintas, and Isabelle Le Parco for animals' care at the animal core facility "Buffon" of the University of Paris/Jacques Monod Institute. We also thank Flore Torsy for assistance with real-time quantitative PCR.

Funding agencies: This work was funded by a grant ANR-16-CE14-0026 (fat4brain) to $H G$ and $C M$. HS and MM are funded by a PhD fellowship from the French Ministry of Research.

Disclosure: The authors declared no conflict of interest.

Author contributions: HS, MM, GT, ML, and NK carried out experiments and analyzed data. HS, J-SS, PG, CM, FP, and HG conceived the experiments and analyzed data. All authors were involved in writing the paper and had final approval of the submitted version.

Supporting information: Additional Supporting Information may be found in the online version of this article.

\section{References}

1. World Health Organization. Overweight and obesity. Updated April 1, 2020. Accessed July 17, 2020. https://www.who.int/news-room/fact-sheets/detail/obesity-and-overw eight

2. Goossens GH. The metabolic phenotype in obesity: fat mass, body fat distribution, and adipose tissue function. Obes Facts 2017;10:207-215.

3. Sorop O, Olver TD, van de Wouw J, et al. The microcirculation: a key player in obesity-associated cardiovascular disease. Cardiovasc Res 2017;113:1035-1045.

4. Kopelman PG. Obesity as a medical problem. Nature 2000;404:635-643.

5. Iadecola $\mathrm{C}$. The neurovascular unit coming of age: a journey through neurovascular coupling in health and disease. Neuron 2017;96:17-42.

6. Roger VL, Go AS, Lloyd-Jones DM, et al. Heart disease and stroke statistics-2011 update: a report from the American Heart Association. Circulation 2011;123:e18-e209.

7. Rhea EM, Salameh TS, Logsdon AF, Hanson AJ, Erickson MA, Banks WA. Bloodbrain barriers in obesity. AAPS J 2017;19:921-930.

8. Haley MJ, Krishnan S, Burrows D, et al. Acute high-fat feeding leads to disruptions in glucose homeostasis and worsens stroke outcome. J Cereb Blood Flow Metab 2019;39:1026-1037.

9. Maysami S, Haley MJ, Gorenkova N, Krishnan S, McColl BW, Lawrence CB. Prolonged diet-induced obesity in mice modifies the inflammatory response and leads to worse outcome after stroke. J Neuroinflammation 2015;12:140. doi:10.1186/s1297 4-015-0359-8

10. Li W, Prakash R, Chawla D, et al. Early effects of high-fat diet on neurovascular function and focal ischemic brain injury. Am J Physiol Regul Integr Comp Physiol 2013;304:R1001-R1008.

11. Gustafson DR, Bäckman K, Joas E, et al. 37 years of body mass index and dementia: observations from the prospective population study of women in Gothenburg, Sweden. J Alzheimers Dis 2012;28:163-171.

12. Beydoun MA, Beydoun HA, Wang Y. Obesity and central obesity as risk factors for incident dementia and its subtypes: a systematic review and meta-analysis. Obes Rev 2008;9:204-218.

13. Pétrault O, Pétrault M, Ouk T, Bordet R, Bérézowski V, Bastide M. Visceral adiposity links cerebrovascular dysfunction to cognitive impairment in middle-aged mice. Neurobiol Dis 2019;130:104536. doi:10.1016/j.nbd.2019.104536 
14. Coucha M, Abdelsaid M, Ward R, Abdul Y, Ergul A. Impact of metabolic diseases on cerebral circulation: structural and functional consequences. Compr Physiol 2018;8:773-799.

15. Tucsek Z, Toth P, Tarantini S, et al. Aging exacerbates obesity-induced cerebromicrovascular rarefaction, neurovascular uncoupling, and cognitive decline in mice. $J$ Gerontol A Biol Sci Med Sci 2014;69:1339-1352.

16. Schaefer AT, Margrie TW. Spatiotemporal representations in the olfactory system. Trends Neurosci 2007;30:92-100.

17. Osmanski BF, Martin C, Montaldo G, et al. Functional ultrasound imaging reveals different odor-evoked patterns of vascular activity in the main olfactory bulb and the anterior piriform cortex. NeuroImage 2014;95:176-184.

18. Pain F, L'Heureux B, Gurden H. Visualizing odor representation in the brain: a review of imaging techniques for the mapping of sensory activity in the olfactory glomeruli. Cell Mol Life Sci 2011;68:2689-2709.

19. Otsu Y, Couchman K, Lyons DG, et al. Calcium dynamics in astrocyte processes during neurovascular coupling. Nat Neurosci 2015;18:210-218.

20. Petzold GC, Albeanu DF, Sato TF, Murthy VN. Coupling of neural activity to blood flow in olfactory glomeruli is mediated by astrocytic pathways. Neuron 2008;58:897-910

21. Gurden H, Uchida N, Mainen ZF. Sensory-evoked intrinsic optical signals in the olfactory bulb are coupled to glutamate release and uptake. Neuron 2006;52:335-345.

22. Rungta RL, Chaigneau E, Osmanski B-F, Charpak S. Vascular compartmentalization of functional hyperemia from the synapse to the pia. Neuron 2018;99:362-375.e4.

23. Soleimanzad H, Smekens F, Peyronnet J, et al. Multiple speckle exposure imaging for the study of blood flow changes induced by functional activation of barrel cortex and olfactory bulb in mice. Neurophotonics 2019;6:015008. doi:10.1117/1.NPh.6.1.015008

24. Kazmi SMS, Faraji E, Davis MA, Huang Y-Y, Zhang XJ, Dunn AK. Flux or speed? Examining speckle contrast imaging of vascular flows. Biomed Opt Express 2015;6:2588-2608

25. Schrandt CJ, Kazmi SMS, Jones TA, Dunn AK. Chronic monitoring of vascular progression after ischemic stroke using multiexposure speckle imaging and two-photon fluorescence microscopy. J Cereb Blood Flow Metab 2015;35:933-942.

26. Paxinos G, Franklin KBJ. Paxinos and Franklin's the Mouse Brain in Stereotaxic Coordinates. Academic Press; 2019.

27. Parthasarathy AB, Tom WJ, Gopal A, Zhang X, Dunn AK. Robust flow measurement with multi-exposure speckle imaging. Opt Express 2008;16:1975-1989.

28. Renier N, Wu Z, Simon DJ, Yang J, Ariel P, Tessier-Lavigne M. iDISCO: a simple, rapid method to immunolabel large tissue samples for volume imaging. Cell 2014;159:896-910.

29. Mortreux M, Foppen E, Denis RG, et al. New roles for prokineticin 2 in feeding behavior, insulin resistance and type 2 diabetes: studies in mice and humans. Mol Metab 2019;29:182-196

30. Wierschke S, Gigout S, Horn P, et al. Evaluating reference genes to normalize gene expression in human epileptogenic brain tissues. Biochem Biophys Res Commun 2010;403:385-390.

31. Yang M, Crawley JN. Simple behavioral assessment of mouse olfaction. Curr Protoc Neurosci 2009; 48:8.24.1-8.24.12.

32. Duarte JV, Pereira JMS, Quendera B, et al. Early disrupted neurovascular coupling and changed event level hemodynamic response function in type 2 diabetes: an fMRI study. J Cereb Blood Flow 2015;35:1671-1680.
33. Panczel G, Daffertshofer M, Ries S, Spiegel D, Hennerici M. Age and stimulus dependency of visually evoked cerebral blood flow responses. Stroke 1999;30:619-623.

34. Karason K, Wallentin I, Larsson B, Sjöström L. Effects of obesity and weight loss on cardiac function and valvular performance. Obes Res 1998;6:422-429.

35. Shiba K, Machida T, Uchida S, Hotta H. Sympathetic neural regulation of olfactory bulb blood flow in adult and aged rats. Auton Neurosci 2009;147:75-79.

36. Toth P, Tarantini S, Csiszar A, Ungvari Z. Functional vascular contributions to cognitive impairment and dementia: mechanisms and consequences of cerebral autoregulatory dysfunction, endothelial impairment, and neurovascular uncoupling in aging. Am J Physiol Heart Circ Physiol 2017;312:H1-H20.

37. Tarantini S, Valcarcel-Ares MN, Yabluchanskiy A, et al. Nrf2 deficiency exacerbates obesity-induced oxidative stress, neurovascular dysfunction, blood-brain barrier disruption, neuroinflammation, amyloidogenic gene expression, and cognitive decline in mice, mimicking the aging phenotype. J Gerontol A Biol Sci Med Sci 2018;73:853-863.

38. Li Y, Choi WJ, Wei W, et al. Aging-associated changes in cerebral vasculature and blood flow as determined by quantitative optical coherence tomography angiography. Neurobiol Aging 2018;70:148-159.

39. Fu Z, Wu J, Nesil T, Li MD, Aylor KW, Liu Z. Long-term high-fat diet induces hippocampal microvascular insulin resistance and cognitive dysfunction. Am J Physiol Endocrinol Metab 2017;312:E89-E97.

40. Hinkel R, Howe A, Renner S, et al. Diabetes Mellitus-Induced Microvascular Destabilization in the Myocardium. J Am Coll Cardiol 2017;69:131-143.

41. Spinetti G, Kraenkel N, Emanueli C, Madeddu P. Diabetes and vessel wall remodelling: from mechanistic insights to regenerative therapies. Cardiovasc Res 2008;78:265-273.

42. Ungvari Z, Tarantini S, Donato AJ, Galvan V, Csiszar A. Mechanisms of vascular aging. Circ Res 2018;123:849-867.

43. Ye J. Mechanisms of insulin resistance in obesity. Front Med 2013;7:14-24.

44. Hoscheidt SM, Kellawan JM, Berman SE, et al. Insulin resistance is associated with lower arterial blood flow and reduced cortical perfusion in cognitively asymptomatic middle-aged adults. J Cereb Blood Flow Metab 2017;37:2249-2261.

45. Toth P, Tarantini S, Ashpole NM, et al. IGF-1 deficiency impairs neurovascular coupling in mice: implications for cerebromicrovascular aging. Aging Cell 2015;14:1034-1044.

46. Aimé $P$, Hegoburu C, Jaillard T, et al. A physiological increase of insulin in the olfactory bulb decreases detection of a learned aversive odor and abolishes food odor-induced sniffing behavior in rats. PLoS One 2012;7:e51227. doi:10.1371/journal.pone.0051227

47. Edwin Thanarajah S, Hoffstall V, Rigoux L, Hanssen R, Brüning JC, Tittgemeyer M. The role of insulin sensitivity and intranasally applied insulin on olfactory perception. Sci Rep 2019;9:7222. doi:10.1038/s41598-019-43693-7

48. Hinds JW, McNelly NA. Capillaries in aging rat olfactory bulb: a quantitative light and electron microscopic analysis. Neurobiol Aging 1982;3:197-207.

49. Villena A, Vidal L, Díaz F, Pérez De Vargas I. Stereological changes in the capillary network of the aging dorsal lateral geniculate nucleus. Anat Rec A Discov Mol Cell Evol Biol 2003;274:857-861.

50. Burns EM, Kruckeberg TW, Gaetano PK. Changes with age in cerebral capillary morphology. Neurobiol Aging 1981;2:283-291.

51. Ronan L, Alexander-Bloch AF, Wagstyl K, et al. Obesity associated with increased brain age from midlife. Neurobiol Aging 2016;47:63-70.

52. Aanerud J, Borghammer P, Chakravarty MM, et al. Brain energy metabolism and blood flow differences in healthy aging. J Cereb Blood Flow Metab 2012;32:1177-1187.

53. Dutta S, Sengupta P. Men and mice: relating their ages. Life Sci 2016;152:244-248. 